\title{
Foods of Bats (Family Vespertilionidae) at Five Locations in New Hampshire and Massachusetts
}

\author{
Howard H. Thomas ${ }^{1}$, Paul R. Moosman, Jr. ${ }^{2}$, Jacques Pierre Veilleux ${ }^{3}$, and Jason Holt ${ }^{1,4}$ \\ ${ }^{1}$ Department of Biology and Chemistry, Fitchburg State University, Fitchburg, Massachusetts 01420 USA \\ ${ }^{2}$ Department of Biology, Virginia Military Institute, Lexington, Virginia 24450 USA \\ ${ }^{3}$ Department of Biology, Franklin Pierce University, Rindge, New Hampshire 03461 USA \\ ${ }^{4}$ Current address: 19 Railroad Street, Acton, Massachusetts 01720 USA
}

Thomas, Howard H., Paul R. Moosman, Jr., Jacques Pierre Veilleux, and Jason Holt. 2012. Foods of bats (Family Vespertilionidae) at five locations in New Hampshire and Massachusetts. Canadian Field-Naturalist 126(2): 117-124.

Diet and feeding relations of six species of bats at five locations in New Hampshire and Massachusetts were studied to improve understanding of foraging niche differentiation. Fecal samples were collected from 100 Big Brown Bats (Eptesicus fuscus), 154 Little Brown Myotis (Myotis lucifugus), 49 Northern Myotis (M. septentrionalis), 54 Eastern Small-footed Myotis (M. leibii), 9 Eastern Red Bats (Lasiurus borealis), and 1 Hoary Bat (L. cinereus) netted during non-hibernation periods from 2004 to 2008 at four locations in southern New Hampshire and one in north-central Massachusetts. Beetles (Order Coleoptera) were the major food of E. fuscus (mean percentage volume $=81.6 \%, 97 \%$ occurrence) followed by moths (Order Lepidoptera), with scarabaeid and carabid beetles the most abundant consumed families by volume and frequency. Moths were the most important item by volume and frequency preyed on by the remaining species (M. lucifugus, mean percentage volume $30.7 \%$, $82 \%$ occurrence; $M$. septentrionalis, mean percentage volume $42.7 \%$, $82 \%$ occurrence; $M$. leibii, mean percentage volume $49.4 \%, 81 \%$ occurrence; $L$. borealis, mean percentage volume $62.8 \%, 100 \%$ occurrence; $L$. cinereus, mean percentage volume $82 \%, 100 \%$ occurrence). Little Brown Myotis consumed the largest variety of prey (40); Northern Myotis consumed the highest volume of spiders $(8.1 \%)$. Community similarity index values indicated diets of the three species of Myotis were more similar to each other (similarity $=0.71$ ) than to those of non-Myotis. The diet of E. fuscus was more similar to that of the Myotis cluster (0.58) than to either species of Lasiurus. Results suggest that, despite faunal differences between the U.S. Northeast and other parts of North America, foraging relationships among guild members follows a similar pattern.

Key Words: Big Brown Bat, Eptesicus fuscus, Little Brown Myotis, Myotis lucifugus, Northern Myotis, Myotis septentrionalis, Eastern Small-footed Myotis, Myotis leibii, Eastern Red Bat, Lasiurus borealis, Hoary Bat, Lasiurus cinereus, Lepidoptera, scarabaeid beetle, carabid beetle, New Hampshire, Massachusetts.

The role of resource partitioning in feeding guilds of insectivorous bats has been examined by a number of authors (e.g. Black 1974, Carter et al. 2003, Whitaker 2004, Feldhamer et al. 2009, Moosman et al. 2012). The diets of temperate insectivorous bats are closely related to factors such as foraging habitat (Aldridge and Rautenbach 1987; Furlonger et al. 1987), climate (Moosman et al. 2012), prey hardness (Aldridge and Rautenbach 1987; Furlonger et al. 1987), characteristics of prey detection systems (Barclay and Brigham 1991), cranial and wing morphology (Belwood and Fenton 1976; Freeman 1981), and jaw musculature (Aldridge and Rautenbach 1987; Furlonger et al. 1987). Since bat species have adapted to these factors differently their diet may reflect such adaptations (Aldridge and Rautenbach 1987).

Most authors have defined guild structure according to differential prey utilization (Whitaker 1972; Kunz 1973; Black 1974; Whitaker et al. 1981; Griffith and Gates 1985; Warner 1985; Carter et al. 2003; Whitaker 2004; and Feldhamer et al. 2009). However, comparing results across studies is difficult because of variation in both bat diet and study methodology. Geographic dietary variation has been detected in well-studied species such as Eptesicus fuscus (Big Brown Bat) and Myotis lucifugus (Little Brown Myotis) (Moosman et al. 2012). Studies in western North America reported relatively low volumes of coleopterans in the diet of Big Brown Bats, a species typically considered a coleopteran specialist (Freeman 1981). Additionally, Whitaker and Lawhead (1992) reported high volumes of lepidopterans $(71 \%)$ relative to other studies, in the diet of the generalist Little Brown Myotis.

Some authors described diets of bats using percentage volume (e. g. Whitaker 2004) whereas others used frequency of occurrence, or estimated number of insects consumed (e.g. Black 1974; Brigham and Saunders 1990). More recently, Whitaker (2004) and Feldhamer et al. (2009) described insectivorous bat feeding guilds by examining relatedness of diets of bat species in a guild. Both studies utilized community similarity and diversity indices to examine the feeding guild relationships of bat communities in the northern contiguous U.S., with Whitaker (2004) reporting on bats from Indiana and Feldhamer et al. (2009) on bats from Illinois.

The bat community of northeastern North America consists of nine species with the genus Myotis accounting for the largest portion (three species). In both northern Massachusetts and southern New Hampshire the community typically consists of six or seven species: Big Brown Bat (Eptesicus fuscus), Eastern Red Bat (Lasiurus borealis), Hoary Bat (L. cinereus), Northern 
Myotis (Myotis septentrionalis), Little Brown Myotis (M. lucifugus), Eastern Small-footed Myotis (M. leibii), and Tricolored Bat (Perimyotis subflavus). The presence of Eastern Small-footed Myotis and the rarity of the Silver-haired Bat (Lasionycteris noctivagans) in northeastern North America make this particular community unique among dietary studies, as does its lack of Indiana Myotis (Myotis sodalis). Although others have examined the diets of bat communities containing some of the same species in other localities (e.g., Griffith and Gates 1985; Carter et al. 2003; Whitaker 2004; and Feldhamer et al. 2009) there have been no comprehensive dietary studies of bat communities in New England.

The purpose of this study was to identify prey eaten by bats from north-central Massachusetts and southern New Hampshire, to examine the similarity of prey consumed by bats in this community, and compare our results to those of other studies. This dietary study represents the only one to examine a community of bats that includes the Eastern Small-footed Myotis, which is currently under review to be listed under the U.S. Endangered Species Act.

\section{Study Area and Methods}

\section{Study areas}

Bats were netted, between 2004 and 2008 (May to September in 2004 and 2005, May to October in 2006 and 2007, and April to July 2008) at the following locations in southern New Hampshire and north-central Massachusetts.

New Hampshire-Cheshire County, Pisgah State Park, located in three townships (Chesterfield, Hinsdale, and Winchester), $42^{\circ} 50^{\prime} \mathrm{N}$, $72^{\circ} 26^{\prime} \mathrm{W}$; Cheshire County, Surry Mountain Dam, Surry Township, $43^{\circ} 00^{\prime} \mathrm{N}, 72^{\circ} 19^{\prime} \mathrm{W}$; Hillsborough County, Edward MacDowell Dam, Peterborough Township, 42 54 'N, $71^{\circ} 58^{\prime} \mathrm{W}$; Hillsborough County, New Boston Air Force Station, New Boston Township, $42^{\circ} 56^{\prime} \mathrm{N}, 71^{\circ} 38^{\prime} \mathrm{W}$. Massachusetts-Worcester County, Mt. Watatic, Ashburnham Township, $42^{\circ} 42^{\prime} \mathrm{N}, 71^{\circ} 54^{\prime} \mathrm{W}$.

Pisgah State Park is undeveloped and consists of $>5380$ ha (Veilleux et al. 2008). The landscape is dominated by coniferous, mixed conifer/deciduous and deciduous forests. Most forests are second growth but there are three areas of old growth remnants inside the park. Surry Mountain Lake is an impoundment of the Ashuelot River created by the Surry Mountain Dam, built by the U.S. Army Corps of Engineers. Topography surrounding Surry is steep. In addition to the Surry Mountain Lake (reservoir) and the Ashuelot River proper, there are several oxbow wetlands downstream from the dam. The site has mixed deciduous and coniferous forest types. Edward MacDowell Reservoir is an impoundment of Nubanusitt Brook maintained by the U.S. Army Corps of Engineers. The resulting lake is relatively shallow and as such has extensive emergent vegetation along its periphery. The surrounding forest is mostly mixed deciduous and coniferous. New Boston Air Force Station is predominantly forested. Its topography is dominated by rolling terrain. Surrounding forest is mostly mixed deciduous. Mt. Watatic is a prominent terrain feature in north-central Massachusetts just south of the New Hampshire state line, with slopes of mixed deciduous forest.

\section{Capture of bats}

Bats were captured using mist nets. Nets were placed across access roads and less often across woodland streams or rivers, or perpendicular to the edges of grassy openings. Following capture each bat was identified and then placed in an individual cloth bag for approximately 20 minutes to collect fecal pellets. Fecal pellets were stored in plastic vials. Bats were released at their site of capture. Methods used to handle bats followed guidelines of the American Society of Mammalogists (Sikes et al. 2011) and were approved by the Animal Care Committee of Fitchburg State University (Fitchburg, Massachusetts).

\section{Sample analysis and prey identification}

Fecal pellets were wetted with $70 \%$ isopropyl alcohol and teased apart using dissecting tools while being viewed through a dissecting microscope. All pellets from a single bat were pooled as a single sample. Prey items were identified to order and family (when possible) using a reference collection of arthropods from the sampling locations as well as elsewhere in the region.

The percentage volume of each prey item for each sample was visually estimated and the mean percentage volume for each prey item was calculated for each bat species following methods of Whitaker (1988), which have been widely used across dietary studies. Visual estimation of volume has been the most commonly used method to quantify diet (Moosman et al. 2012) and it was used to facilitate comparison with the greatest number of studies (Whitaker 1972; Whitaker et al. 1981; Griffith and Gates 1985; Whitaker and Lawhead 1992; Whitaker 1995; Agosta and Morton 2003; Carter et al. 2003; Whitaker 2004; Whitaker and Barnard 2005; Johnson and Gates 2007; Moosman et al. 2007; Feldhamer et al. 2009).

Additionally, percentage occurrence of each prey item was calculated for each bat species. A list of prey items with respective mean percentage volume and percentage occurrence values was developed for each bat species (Table 1), and comparisons of prey community similarities were made using Jaccard Similarity Coefficients (Sneath and Sokal 1973). This allowed comparison of our data with those obtained by Whitaker (2004) and Feldhammer et al. (2009).

\section{Results}

During 26 months of sampling, a total of 367 bat fecal samples were collected. The number of samples 


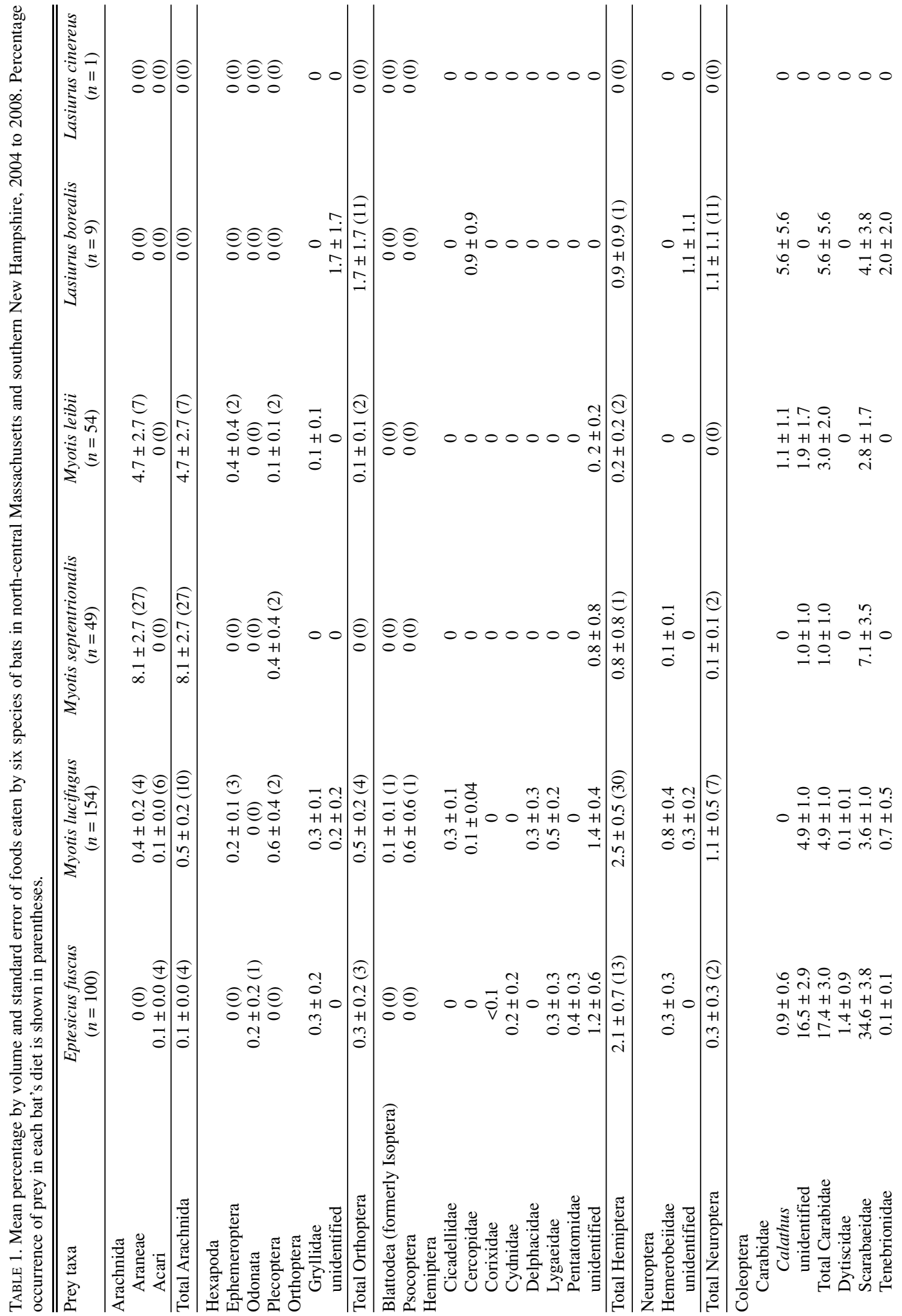




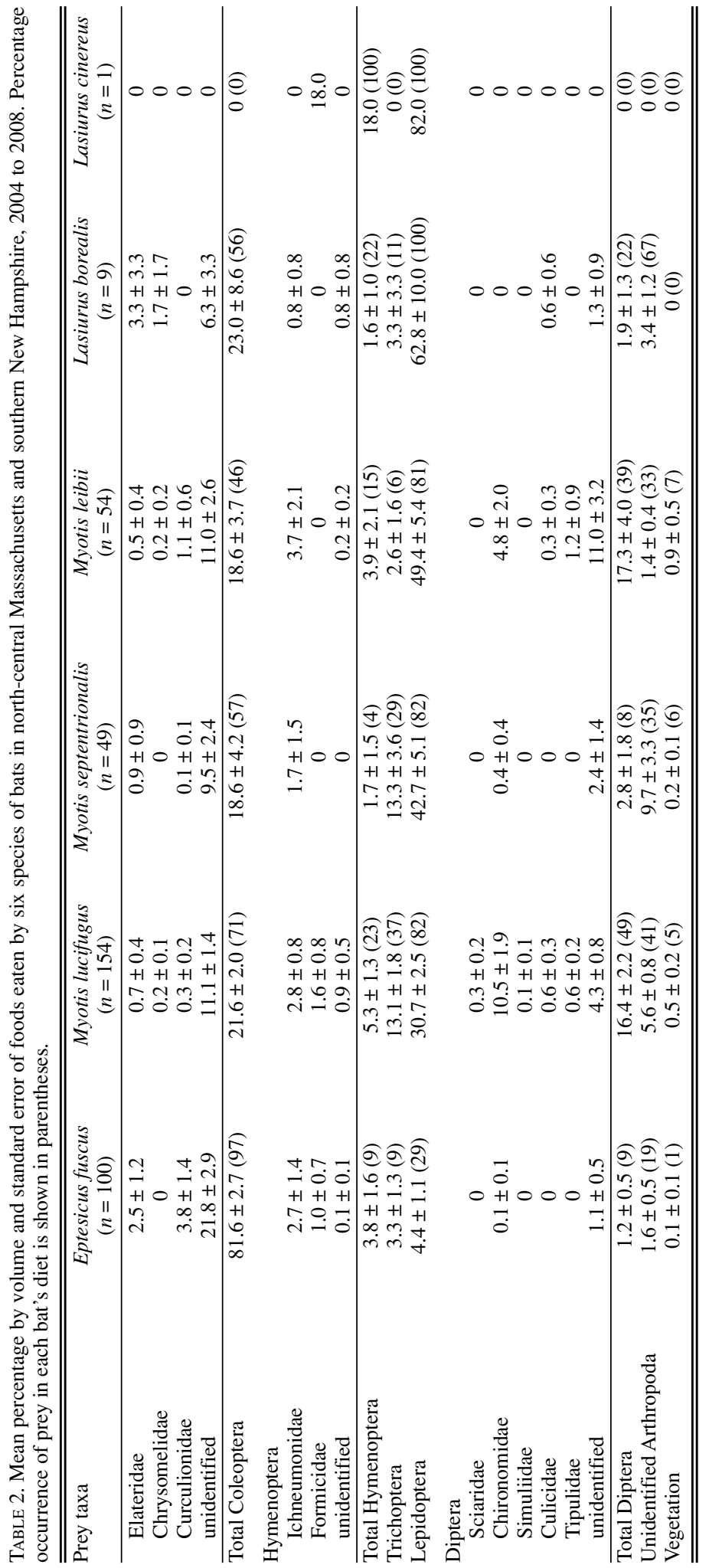


collected from each site was as follows: 194 from Surry Mountain Dam, 87 from Pisgah State Park, 42 from Edward MacDowell Lake, 36 from New Boston Air Force Station and the one from Mt. Watatic. Samples from Eptesicus fuscus, Lasiurus borealis, Myotis lucifugus, and M. septentrionalis were collected from most (three or four of the five) sites. However, samples from M. leibii were restricted to Surry Mountain Dam and New Boston as Air Force Station and the one from $L$. cinereus were restricted to New Boston Air Force Station.

\section{Prey consumed \\ Eptesicus fuscus $(\mathrm{n}=100)$.}

Big Brown Bats fed primarily on seven families of beetles (Order Coleoptera). Beetles occurred in the diet of 97of 100 samples examined (97\%) and constituted the greatest volume of food consumed (mean percentage volume and standard error are reported: $81.6 \% \pm 2.7$ ) (Table 1). In most samples a portion of the beetle material reported was identifiable only to the ordinal level $(21.8 \% \pm 2.9)$. Of the beetle material identified to the family level those in Scarabaeidae and Carabidae were the most prominent. Less often consumed were beetles of the families Curculionidae, Elateridae, Dytiscidae, and Tenebrionidae (Table 1). The carabid beetle genus Calathus was identified in the foods of 3 of 100 Big Brown Bats and represented a mean volume of $0.9 \% \pm 0.6$. Moths (Order Lepidoptera) were the second most commonly consumed arthropods occurring in $29 \%$ of the samples and a mean percentage volume of $4.4 \% \pm 1.1$ (Table 1). This was followed by the orders Hymenoptera and Hemiptera, unidentified insect remains, and the order Diptera. Remaining prey were of the orders Acari, Trichoptera, Orthoptera, Neuroptera, and Odonata.

\section{Myotis lucifugus $(\mathrm{n}=154)$}

Little Brown Myotis from our study fed prominently on lepidopteran insects (Table 2). Lepidopterans occurred most frequently $(82 \%)$ and represented the greatest volume $(30.7 \% \pm 2.5)$ in the diet of this bat. This species also utilized the widest array of prey in this study. Other important prey ( $>1 \%$ volume) were in descending order: Coleoptera, Diptera, Trichoptera, Hymenoptera, Hemiptera, and Neuroptera. Each of the above orders was similarly arranged in descending order of frequency of occurrence. Occurring in lesser volumes $(<1 \%)$ were prey from Araneae, Acari, Orthoptera, Ephemeroptera, Plecoptera, Blattodea (formerly Isoptera), and Psocoptera. The following families within the above orders were identified: seven families of Coleoptera, five families of Diptera, four families of Hemiptera, two families of Hymenoptera, and a single family of the order Orthoptera was also found (Table 1).

Myotis septentrionalis $(\mathrm{n}=49)$

Northern Myotis had a diet similar to Little Brown Myotis with lepidopteran insects representing the high- est occurrence (82\%) and highest mean percentage volume $(42.7 \% \pm 5.1)$. Other prey occurring with a mean percentage volume of $\geq 1 \%$ were: scarabaeid and carabid beetles as well as unidentified Coleoptera, Trichoptera, Araneae, Diptera, and Hymenoptera. Chironomids and Ichneumonid wasps were the only family of dipterans and hymenopterans identified, respectively. Northern Myotis consumed the highest amount of spiders of the species studied (Table 1) in line with their gleaning behavior.

\section{Myotis leibii $(\mathrm{n}=54)$}

Foods eaten by the Eastern Small-footed Myotis in our study area were previously reported (Moosman et al. 2007) but we have incorporated an additional 15 samples. As with the other two species of the genus Myotis, Eastern Small-footed Myotis consumed moths at both high frequency of occurrence and volume (Table 1). After moths, Coleoptera and Diptera were the two most common prey items eaten by the Eastern Smallfooted Myotis (Table 1). Of prey items identified to family, chironomid flies, ichneumonid wasps, small scarabaeid, carabid and curculionid beetles, and crane flies (Tipulidae) were the most abundant components (Table 1).

\section{Lasiurus borealis $(\mathrm{n}=9)$}

The Eastern Red Bat fed most frequently on lepidopteran (100\%) and coleopteran (56\%) insects (Table $1)$. Both of the above orders of insects also represented the greatest volumes in this bat's diet, with lepidopterans accounting for a mean percentage volume of $62.8 \% \pm 10.0$ and coleopterans $23.0 \% \pm 8.6$. Other insect prey found in volumes $\geq 1 \%$ included trichopterans, dipterans, orthopterans, hymenopterans, and neuropterans. Hemipteran insects constituted $0.9 \% \pm 0.9$ of the volume of the fecal sample from one Eastern Red Bat. Coleopteran families identified as prey included Scarabaeidae, Carabidae (including the genus Calathus), Chrysomelidae, Tenebrionidae, and Elateridae. Scarabaeid beetles accounted for only $3 \%$ of the volume in the single sample in which they were found, elaterid beetles represented $30 \%$ of the volume of the one sample in which they were found, and the carabid beetle genus Calathus accounted for $50 \%$ of the volume of the single sample in which it was found (Table 1).

\section{Lasiurus cinereus $(\mathrm{n}=1)$}

The single Hoary Bat sample from our study revealed that $82 \%$ of the volume was made up of moths and the remaining volume consisted of hymenopterous insects (Table 1).

\section{Prey Community Similarities}

All species of bats reported showed a strong tendency to feed on terrestrial insects (i.e., beetles, moths, most of the dipterans, and hymenopterans). All three species of the genus Myotis that occurred in the community of bats at our study sites had diets that were relatively similar (Figure 1) (community similarity value 
among the three species of 0.71). The diets of Eastern Small-footed and Little Brown Myotis were even more similar (0.78). The diets of the three species of Myotis were more similar to each other than to those of other guild members. Dependence on beetles in the diet of the Big Brown Bat made its diet distinct from those of the three species of Myotis but more similar to them than to the diets of Eastern Red Bat or Hoary Bat (Table 1). The similarity value of the Big Brown Bat's diet (0.58) places it between the cluster of Myotis and the Eastern Red Bat (Figure 1). The two Lasiurus species were relatively distinct in comparison to the other species in the guild (Figure 1). The diet of the Eastern Red Bat was more similar to the diet of the Big Brown Bat than to the diet of the Hoary Bat.

\section{Discussion}

Results of the present study add to a limited body of information about food habits of bats from New England. Anthony and Kunz (1977) presented information on foods eaten by Little Brown Myotis in New Hampshire. Moosman et al. (2007) provided some of the first records for foods of the Eastern Small-footed Myotis, and Moosman et al. (2012) reported the four most used categories of food eaten by Little Brown Myotis and the Big Brown Bat in this region in context of the diet of these species throughout their ranges. Our findings represent the first published records of foods for the Northern Myotis, Eastern Red Bat, and Hoary Bat in New England. Our results show how relationships among guild members compare to patterns reported from other North American bat communities.

\section{Eptesicus fuscus}

Our data indicated that Big Brown Bats preyed on beetles most frequently and in the highest proportion of volume of any prey items, and our data are in agreement with reports by most other investigators (Hamilton 1933; Whitaker et al. 1981; Griffith and Gates 1985; Brigham and Saunders 1990; Whitaker 1995; Agosta and Morton 2003; Carter et al. 2003; Whitaker 2004; Feldhamer et al. 2009). The predominance of beetles is in keeping with the recognized niche of this bat as a beetle specialist (Freeman 1981) or strategist (Black 1974). High incidence and volume of beetles of the families Scarabaeidae and Carabidae are likewise similar to values reported by Whitaker (1972) and Griffith and Gates (1985). However, moths were the second most important prey item of Big Brown Bats in our study, and this finding differs from most other studies. These differences may have been at least partially influenced by climatic differences between sites or by the time of year the bats were netted. Moosman et al. (2012) offered evidence that moths are used less often by big brown bats in regions with particularly high summer precipitation, such as New England.

\section{Myotis species}

Little Brown Myotis in New Hampshire consumed moths most frequently and at the highest percentage

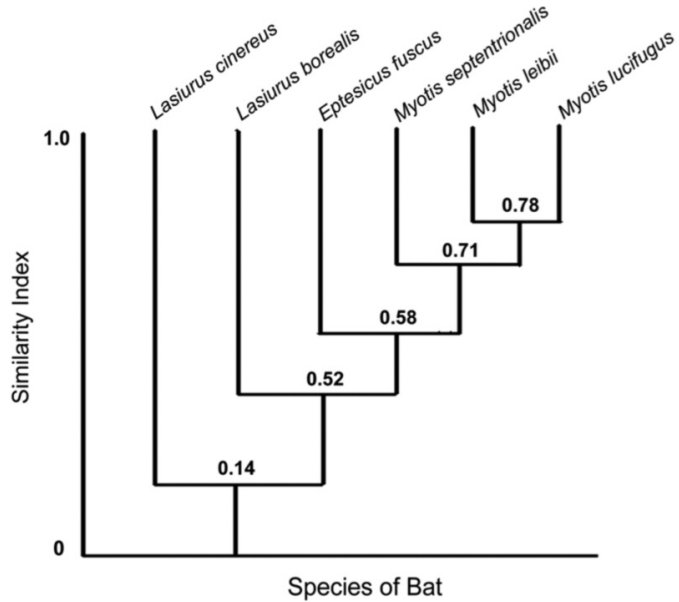

FIGURE 1. Similarity of diets of six species of bats in New England between 2004 and 2008, based on Jaccard similarity coefficient values.

volume of all food items. These results are similar to those reported from Indiana (Whitaker 1972), New Hampshire (Anthony and Kunz 1977), and West Virginia (Carter et al. 2003). Moths were second to beetles in the diet of Little Brown Myotis from western Maryland (Griffith and Gates 1985). However, our results contrast to those of Whitaker (2004) in which moths were found at a lower volume compared to dipterans in the diet of the Little Brown Myotis in Indiana. Our results are similar to those found by Carter et al. (2003), in which the descending percent volume order was Diptera, Trichoptera, Coleoptera, Hymenoptera, and Homoptera.

Our data showed that Northern Myotis fed heavily on moths. These results are similar to those reported by Whitaker (1972) and Griffith and Gates (1985) but in contrast to Carter et al. (2003) and Whitaker (2004). The next most frequently eaten arthropod by Northern Myotis in New Hampshire was beetles. Griffith and Gates (1985) listed beetles as the second most frequently occurring food item for this bat in western Maryland. According to volumetric data, Northern Myotis from New England ate fewer beetles than those in West Virginia (Carter et al. 2003), Indiana (Whitaker 2004), or southern Illinois (Feldhamer et al. 2009).

Remaining prey taxa are similar to other studies with two exceptions. Whitaker (2004) reported dipterans as having the highest percentage volumes in the diet of Northern Myotis from Indiana. Whitaker (2004) and Feldhamer et al. (2009) contain the only other reports of spiders in the diet of this species. Whitaker (2004) reported a lower volume of spiders $(2.0 \%)$ while Feldhamer et al. (2009) reported higher volumes (15.6\%). Our mean percentage volume was $8.1 \%$. Dietary differences between Northern Myotis from Indiana and New Hampshire likely reflect differences in habitat. 
Whereas Indiana is primarily an agricultural state, New Hampshire has minimal agriculture and is heavily forested. The Northern Myotis is a forest-dwelling bat, and this suggests why it may encounter spiders in forested sites such as New England more frequently than in the more fragmented agricultural landscapes of Indiana.

\section{Lasiurus species}

The diet of the Eastern Red Bat reported here is similar to that reported elsewhere. The predominance, by volume and occurrence, of moths and beetles has been reported for Eastern Red Bats in West Virginia (Carter et al. 2003) and in Indiana (Whitaker 2004). The diet of our single Hoary Bat was similar to that reported elsewhere. Whitaker (1972) reported that the diets two of three Hoary Bats from Indiana consisting of $100 \%$ moths. Five individuals from West Virginia consumed moths at a volume of $98 \%$ (Carter et al. 2003). The remaining volume of our single hoary bat was unidentified Hymenopteran (not ichneumonid wasps). Warner (1985) is the only other study to report hymenopterans in the diet of Hoary Bats.

Dietary similarity within the feeding guild is in agreement with Whitaker (2004) for bats from Indiana and with Feldhamer et al. (2009) for bats from Illinois. The pattern of dietary similarity between Myotis species differs only in that the Indiana Myotis is present in data from Indiana and Illinois, whereas the Eastern Small-footed Myotis is present in the study area reported here. The diet of Big Brown Bats, reported here, is most similar to the myotine bat cluster than either of the lasiurine species. These results are in contrast to those of both Whitaker (2004) and Feldhamer et al. (2009) who found that the Big Brown Bat's diet was most similar to that of the Evening Bat (Nycticeus humeralis) which is absent from the bat community in northern New England.

Differences in diet of bats at our sites relative to other regions could be the result of any number of factors. However, we suspect they reflect the convergence of local climate, prey assemblages, and intraspecific interactions in a bat community with relatively little diversity. Moosman et al. (2012) attributed dietary shifts in such feeding guilds to climate-related differences in the availability of arthropods more than to the diversity of bat species. However, the effects of interspecific competition among insectivorous bats are poorly understood. Thus we encourage authors to report community similarity values in future dietary studies in order to facilitate comparisons across regions.

\section{Acknowledgements}

Our study was supported by U.S. National Science Foundation grants 0754788 and 0330897 . The assistance of the scientists and staff of the U.S. Army Corps of Engineers allowed us access to their lands in Surry and Peterborough Townships. Cooperation of the United State Air Force made it possible to study bats in New
Boston Township. The departments of Fish and Game for Massachusetts and New Hampshire provided us with permits to net bats in various locations in their states, respectively. We recognize the support of K. Austin, C. Cratsley, and L. S. Reynolds for field assistance and providing additional scientific insight. We thank the many undergraduate and graduate students from Fitchburg State University, Franklin Pierce University, and Virginia Military Institute who aided us during many nights in the field.

\section{Literature Cited}

Agosta, S. J., and D. Morton. 2003. Diet of the big brown bat, Eptesicus fuscus, from Pennsylvania and western Maryland. Northeastern Naturalist 10: 89-104.

Aldridge, H. D. J. N., and I. L. Rautenbach. 1987. Morphology, Echolocation and resource partitioning in insectivorous bats. Journal of Animal Ecology 56: 763-778.

Anthony, E. L. P., and T. H. Kunz. 1977. Feeding strategies of the little brown bat, M. lucifugus, in southern New Hampshire. Ecology 58: 775-786.

Barclay, R. M. R., and R. M. Brigham. 1991. Prey detection, dietary niche breadth, and body size in bats: why are aerial insectivorous bats so small? American Naturalist 137: 693-703.

Belwood, J. J., and M. B. Fenton. 1976. Variation in the diet of Myotis lucifugus (Chiroptera: Vespertilionidae). Canadian Journal of Zoology 54: 1674-1678.

Black, H. L. 1974. A north temperate bat community: structure and prey populations. Journal of Mammalogy 55: 138157.

Brigham, R. M., and M. B. Saunders. 1990. The diet of big brown bats (Eptesicus fuscus) in relation to insect availability in southern Alberta, Canada. Northwest Science 64: 7-10.

Carter, T. C., M. A. Menzel, S. F. Owen, J. W. Edwards, J. M. Menzel, and W. M. Ford. 2003. Food habits of seven species of bats in the Allegheny Plateau and Ridge and Valley of West Virginia. Northeastern Naturalist 10: 83-88.

Feldhamer, G. A., T. C. Carter, and J. O. Whitaker, Jr. 2009. Prey consumed by eight species of insectivorous bats from southern Illinois. American Midland Naturalist 162: 43-51.

Freeman, P. W. 1981. Correspondence of food habits and morphology in insectivorous bats. Journal of Mammalogy 62: 166-173.

Furlonger, C. L., H. J. Dewar, and M. B. Fenton. 1987. Habitat use by foraging insectivorous bats. Canadian Journal of Zoology 65: 284-288.

Griffith, L. A., and J. E. Gates. 1985. Food habits of cavedwelling bats in the central Appalachians. Journal of Mammalogy 66: 451-460.

Hamilton, W. J., Jr. 1933. The insect food of the big brown bat. Journal of Mammalogy 14: 155-156.

Johnson, J. B., and J. E. Gates. 2007. Food habits of Myotis leibii during fall swarming in West Virginia. Northeastern Naturalist 14: 317-322.

Kunz, T. H. 1973. Resource utilization: temporal and spatial components of bat activity in central Iowa. Journal of Mammalogy 54: 14-32.

Moosman, P. R., Jr., H. H. Thomas, and J. P. Veilleux. 2007. Food habits of eastern small-footed bats (Myotis 
leibii) in New Hampshire. American Midland Naturalist 158: 354-360.

Moosman, P. R., Jr., H. H. Thomas, and J. P. Veilleux. 2012. Diet of the widespread insectivorous bats Eptesicus fuscus and Myotis lucifugus relative to climate and richness of bat communities. Journal of Mammalogy 93: 491-496.

Sikes, R. S., W. L. Gannon, and The Animal Care and Use Committee of the American Society of Mammalogists. 2011. Guidelines of the American Society of Mammalogists for the use of wild mammals in research. Journal of Mammalogy 92: 235-253.

Sneath, P. H. A., and R. R. Sokal. 1973. Numerical Taxonomy. W. H. Freeman and Co., San Francisco. 573 pages.

Veilleux, J. P., H. H. Thomas, and P. R. Moosman, Jr. 2008. Bats of Pisgah State Park, New Hampshire. Northeastern Naturalist 15: 25-34.

Warner, R. M. 1985. Interspecific and temporal dietary variation in an Arizona bat community. Journal of Mammalogy 66: 45-51.

Whitaker, J. O., Jr. 1972. Food habits of bats from Indiana. Canadian Journal of Zoology 50: 877-883.
Whitaker, J. O., Jr. 1988. Food habits analysis of insectivorous bats. Pages 171-189 in Ecological and behavioral methods for the study of bats. Edited by T. H. Kunz. Smithsonian Institution Press, Washington, D.C.

Whitaker, J. O., Jr. 1995. Food of the big brown bat Eptesicus fuscus from maternity colonies in Indiana and Illinois. American Midland Naturalist 134: 346-350.

Whitaker, J. O., Jr. 2004. Prey selection in a temperate zone insectivorous bat community. Journal of Mammalogy 85: 460-469.

Whitaker, J. O., Jr. and S. M. Barnard. 2005. Food of big brown bats (Eptesicus fuscus) from a colony at Morrow, Georgia. Southeastern Naturalist 4: 111-118.

Whitaker, J. O., Jr., and B. Lawhead. 1992. Foods of Myotis lucifugus in a maternity colony in central Alaska. Journal of Mammalogy 73: 646-648.

Whitaker, J. O., Jr., C. Masser, and S. P. Cross. 1981. Food habits of Eastern Oregon bats, based on stomach and scat analyses. Northwest Science 55: 281-292.

Received 29 January 2012

Accepted 14 June 2012 